TUM/T39-97-31

DFTT $73 / 97$

\title{
NLO Corrections to Deeply-Virtual Compton Scattering ["]
}

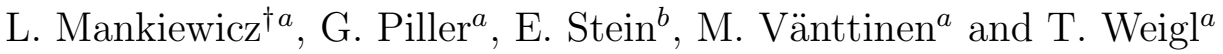 \\ ${ }^{a}$ Physik Department, Technische Universität München, \\ D-85747 Garching, Germany \\ ${ }^{b}$ INFN Sezione di Torino, Via P. Giuria 1, I-10125 Torino, Italy
}

April 4, 2018

\begin{abstract}
:
We have calculated the NLO corrections to the twist-2 part of the deeply-virtual Compton scattering amplitude. Our results for the transverse and antisymmetric parts agree with recent calculations by Ji and Osborne and by Belitsky and Müller. In addition we present NLO results for the longitudinal part of the amplitude.
\end{abstract}

\footnotetext{
*Work supported in part by BMBF

${ }^{\dagger}$ On leave of absence from N. Copernicus Astronomical Center, Polish Academy of Science, ul. Bartycka 18, PL-00-716 Warsaw (Poland)
} 
Our knowledge about the microscopic structure of nucleons is based to a large extent on results from deep-inelastic scattering experiments, which study leading twist parton distribution functions [1]. According to factorization theorems [2] these can be linked to forward matrix elements of twist-2 operators. Forward parton distributions measure the nucleon response to a process where one parton is removed and subsequently inserted back into the target along a light-like distance, without changing its longitudinal momentum. Recently it has been realized that in hard processes where the target nucleon recoils elastically, like in deeply virtual Compton scattering (DVCS) [3, [1] and hard exclusive lepto-production of mesons [4, 5, 6], one can study generalized, nonforward nucleon parton distributions. They describe a situation where the removed parton changes its longitudinal momentum before returning to the nucleon. As in the forward case, it is possible to derive exact relations between these distributions and matrix elements of QCD operators [3, [, 7, 8]. Some of these matrix elements provide an insight into a gauge-invariant decomposition of the nucleon spin between its constituents [3]. In general it has been recognized that nonforward parton distributions provide fundamental information about the nucleon structure and therefore theoretical [3, 4, 7, 9, 10] and experimental [11, 12] studies of their properties will constitute an important part of future strong interaction physics.

In the present paper we calculate NLO $\alpha_{s}$ corrections to deeply-virtual Compton scattering amplitude in the $\overline{\mathrm{MS}}$ scheme. For the unpolarized case such a calculation has been performed first in Ref. [8]. Besides their consequences for future experimental studies of deeply-virtual Compton scattering, NLO corrections are also of theoretical significance. Based on the conformal invariance of one-loop QCD they can be directly determined from the corresponding corrections to the forward scattering amplitude as observed in Ref. [7]. Indeed, in a recent remarkable paper [9] the full NLO corrections have been derived using conformal symmetry arguments. It is gratifying that our results confirm calculations presented in [8, 9]. In addition, we present the NLO correction to the longitudinal part of the amplitude.

In the following we outline a calculation of the deeply-virtual Compton scattering amplitude

$$
\Pi_{\mu \nu}=i \int d^{4} z e^{-i \bar{q} \cdot z}\left\langle P^{\prime}\left|T\left[J_{\mu}(-z / 2) J_{\nu}(z / 2)\right]\right| P\right\rangle
$$

up to order $\alpha_{s}$ in asymmetric kinematics [13, 14, 15] with $q(P)$ and $q^{\prime}\left(P^{\prime}\right)$ being the initial and final photon (nucleon) momenta, respectively. Within twist-2 accuracy we set $P^{2}=P^{\prime 2}=0$. The virtualities of the initial and final photon are given by $-q^{2}=Q^{2}$ and $-q^{\prime 2}=Q^{\prime 2}$. The momentum transfer to the target nucleon is denoted by $\Delta=P-P^{\prime}=$ $q^{\prime}-q$. With the symmetric combinations of initial and final photon and nucleon momenta, $\bar{q}=\frac{1}{2}\left(q+q^{\prime}\right)$ and $\bar{P}=\frac{1}{2}\left(P+P^{\prime}\right)$, we obtain:

$$
-\bar{q}^{2}=\bar{Q}^{2}=\frac{1}{2}\left(Q^{2}+Q^{\prime 2}\right)+\frac{\Delta^{2}}{4} .
$$

In the following we assume that the virtuality $Q^{2}$ of the initial photon is large and allow 
$Q^{\prime 2}$ to vary between 0 and $Q^{2}$. As a consequence, the asymmetry parameter

$$
c=\frac{Q^{2}-Q^{\prime 2}}{Q^{2}+Q^{\prime 2}}
$$

varies between $c=0$ in the symmetric case of deeply-virtual forward Compton scattering, and $c=1$ when the final photon is on-shell, i.e. for $Q^{\prime 2}=0$. In addition, we consider the case $-\Delta^{2} \ll Q^{2}$, and thus neglect the momentum transfer as compared with $\bar{Q}^{2}$. In this limit the symmetric combination of the initial and final target momentum, $\bar{P}$, can be considered massless, and only the component of $\Delta$ proportional to $\bar{P}$ is relevant:

$$
\Delta=2 \xi \bar{P}+\ldots
$$

It is convenient to introduce the symmetric variable $\bar{\omega}=\frac{2 \bar{P} \cdot \bar{q}}{Q^{2}}$, which is related to the conventional Bjorken variable $\omega=\frac{2 P \cdot q}{Q^{2}}$ by

$$
\bar{\omega}=(1+c) \omega-c=\frac{c}{\xi} .
$$

Because of the linear relation between $\omega$ and $\bar{\omega}$, analytical properties of the DVCS amplitude as a function of each variable are closely related. In particular, at fixed $\Delta^{2}=0$, $\Pi_{\mu \nu}$ is a holomorphic function of $\bar{\omega}$ with cuts running from $-\infty$ to -1 and from 1 to $+\infty$. The physical DVCS process corresponds to $\bar{\omega}$ approaching the right-hand cut from above, i.e. $\bar{\omega}>1$ having a positive, infinitesimal imaginary part.

In the actual calculation we follow a method discussed at length in [16]. We use dimensional regularization with $d=4-2 \epsilon$ for both UV and IR divergences which arise in the massless twist-2 kinematics described above. As explained in [16], the only singularities which appear in this approach arise from the renormalization of twist-2 quark and gluon operators which define the parton distribution functions (77). After evaluating the amplitude in $d$-dimensions we have determined the finite part of $\Pi_{\mu \nu}$ according to the $\overline{\mathrm{MS}}$ scheme.

As a consequence of the operator product expansion the DVCS amplitude can be written as [8]:

$$
\begin{aligned}
& \Pi_{\mu \nu}=\left(-g_{\mu \nu}\right)^{T}\left[\sum_{q} e_{q}^{2} \int_{-1}^{1} \frac{d u}{u} F_{q}\left(u, \xi ; \bar{Q}^{2}\right)\left(C_{0}^{S, q}(u \bar{\omega}, \xi \bar{\omega})+a_{s} C_{F} C_{1}^{S, q}(u \bar{\omega}, \xi \bar{\omega})\right)\right. \\
& \left.+\int_{-1}^{1} \frac{d u}{u} G\left(u, \xi ; \bar{Q}^{2}\right) a_{s} C_{1}^{S, g}(u \bar{\omega}, \xi \bar{\omega})\right] \\
& +i \epsilon_{\mu \nu \alpha \beta} \frac{n^{\alpha} n^{* \beta}}{n \cdot n^{*}}\left[\sum_{q} e_{q}^{2} \int_{-1}^{1} \frac{d u}{u} \Delta F_{q}\left(u, \xi ; \bar{Q}^{2}\right)\left(C_{0}^{A, q}(u \bar{\omega}, \xi \bar{\omega})+a_{s} C_{F} C_{1}^{A, q}(u \bar{\omega}, \xi \bar{\omega})\right)\right. \\
& \left.+\int_{-1}^{1} \frac{d u}{u} \Delta G\left(u, \xi ; \bar{Q}^{2}\right) a_{s} C_{1}^{A, g}(u \bar{\omega}, \xi \bar{\omega})\right] \\
& +\left(\bar{P}_{\nu}-q_{\nu} \frac{\bar{P} \cdot \bar{q}}{q^{2}}\right)\left(\bar{P}_{\mu}-q_{\mu}^{\prime} \frac{\bar{P} \cdot \bar{q}}{q^{2}}\right) \frac{\bar{Q}^{2}}{2(\bar{P} \cdot \bar{q})^{2}}
\end{aligned}
$$




$$
\left(1-\bar{\omega}^{2} \xi^{2}\right)\left[\sum_{q} e_{q}^{2} \int_{-1}^{1} \frac{d u}{u} F_{q}\left(u, \xi ; \bar{Q}^{2}\right) a_{s} C_{F} C_{1}^{L, q}(u \bar{\omega}, \xi \bar{\omega})+\int_{-1}^{1} \frac{d u}{u} G\left(u, \xi ; \bar{Q}^{2}\right) a_{s} C_{1}^{L, g}(u \bar{\omega}, \xi \bar{\omega})\right],
$$

with $a_{s}=\frac{\alpha_{s}\left(\bar{Q}^{2}\right)}{2 \pi}$. The transverse metrics tensor

$$
\left(-g_{\mu \nu}\right)^{T}=\frac{n_{\mu} n_{\nu}^{*}+n_{\nu} n_{\mu}^{*}}{n \cdot n^{*}}-g_{\mu \nu}
$$

and the antisymmetric tensor ${ }^{\ddagger} \epsilon_{\mu \nu \alpha \beta} \frac{n^{\alpha} n^{* \beta}}{n \cdot n^{*}}$ are expressed through two light-like vectors $n$ and $n^{*}$ which fulfill $n^{2}=n^{* 2}=0, \bar{P}=\frac{1}{n \cdot n^{*}}(\bar{P} \cdot n) n^{*}$. The nonforward parton distributions $F\left(u, \xi ; \mu^{2}\right), \Delta F\left(u, \xi ; \mu^{2}\right), G\left(u, \xi ; \mu^{2}\right)$ and $\Delta F\left(u, \xi ; \mu^{2}\right)$ are given by the Fourier transforms of nonforward matrix elements of corresponding twist-2 QCD string operators [3, 田, 18] normalized at a scale $\mu^{2}$ :

$$
\begin{aligned}
F_{q}\left(u, \xi ; \mu^{2}\right) & =\frac{1}{2} \int \frac{d \lambda}{2 \pi} e^{i \bar{P} \cdot n \lambda u}\left\langle P^{\prime}\left|\bar{q}\left(-\frac{\lambda n}{2}\right)\left[-\frac{\lambda n}{2} ; \frac{\lambda n}{2}\right] \hat{n} q\left(\frac{\lambda n}{2}\right)\right| P\right\rangle_{z^{2}=0}, \\
\Delta F_{q}\left(u, \xi ; \mu^{2}\right) & =\frac{1}{2} \int \frac{d \lambda}{2 \pi} e^{i \bar{P} \cdot n \lambda u}\left\langle P^{\prime}\left|\bar{q}\left(-\frac{\lambda n}{2}\right)\left[-\frac{\lambda n}{2} ; \frac{\lambda n}{2}\right] \gamma_{5} \hat{n} q\left(\frac{\lambda n}{2}\right)\right| P\right\rangle_{z^{2}=0}, \\
G\left(u, \xi ; \mu^{2}\right) & =\frac{1}{u \bar{P} \cdot n} \int \frac{d \lambda}{2 \pi} e^{i \bar{P} \cdot n \lambda u} n_{\mu} n_{\nu}\left\langle P^{\prime}\left|G^{\mu \alpha}\left(-\frac{\lambda n}{2}\right)\left[-\frac{\lambda n}{2} ; \frac{\lambda n}{2}\right] G_{\alpha}^{\nu}\left(\frac{\lambda n}{2}\right)\right| P\right\rangle_{z^{2}=0}, \\
\Delta G\left(u, \xi ; \mu^{2}\right) & =\frac{1}{u \bar{P} \cdot n} \int \frac{d \lambda}{2 \pi} e^{i \bar{P} \cdot n \lambda u} n_{\mu} n_{\nu}\left\langle P^{\prime}\left|G^{\mu \alpha}\left(-\frac{\lambda n}{2}\right)\left[-\frac{\lambda n}{2} ; \frac{\lambda n}{2}\right] \tilde{G}_{\alpha}^{\nu}\left(\frac{\lambda n}{2}\right)\right| P\right\rangle_{z^{2}=0},
\end{aligned}
$$

with $\hat{n}=n_{\mu} \gamma^{\mu}$, and $\left[-\frac{\lambda n}{2} ; \frac{\lambda n}{2}\right]$ being the path-ordered exponential which ensures gaugeinvariance nonforward distributions.

The easiest way to present our results is to quote them in the unphysical domain $|\bar{\omega}|<1$ where the amplitude (1) is free from imaginary parts arising from the propagation of on-shell states. There, the required coefficient functions in the $\overline{\mathrm{MS}}$ scheme read

$$
\begin{aligned}
C_{0}^{S, q}(u \bar{\omega}, \xi \bar{\omega})= & \frac{u \bar{\omega}}{1-u \bar{\omega}}-\frac{u \bar{\omega}}{1+u \bar{\omega}}, \\
C_{0}^{A, q}(u \bar{\omega}, \xi \bar{\omega})= & \frac{u \bar{\omega}}{1-u \bar{\omega}}+\frac{u \bar{\omega}}{1+u \bar{\omega}}, \\
C_{1}^{S, q}(u \bar{\omega}, \xi \bar{\omega})= & -\frac{9}{2} \frac{u \bar{\omega}}{1-u \bar{\omega}}+\frac{3 u\left(-2 u+\bar{\omega} u^{2}+\bar{\omega} \xi^{2}\right)}{2(1-\bar{\omega} u)\left(u^{2}-\xi^{2}\right)} \log (1-\bar{\omega} u) \\
& +\frac{u\left(1+\bar{\omega}^{2} u^{2}-2 \bar{\omega}^{2} \xi^{2}\right)}{2 \bar{\omega}(1-\bar{\omega} u)\left(u^{2}-\xi^{2}\right)} \log ^{2}(1-\bar{\omega} u) \\
& +\frac{3 u^{2}\left(1-\bar{\omega}^{2} \xi^{2}\right)}{\left(1-\bar{\omega}^{2} u^{2}\right)\left(u^{2}-\xi^{2}\right)} \log (1-\bar{\omega} \xi)
\end{aligned}
$$

\footnotetext{
${ }^{\ddagger}$ We use the convention of [17] for the epsilon tensor.
} 


$$
\begin{aligned}
& +\frac{u^{2}(1-\bar{\omega} \xi)\left(\bar{\omega}^{2} u^{2}-2 \bar{\omega} \xi-2 \bar{\omega}^{2} \xi^{2}-1\right)}{2 \bar{\omega}\left(1-\bar{\omega}^{2} u^{2}\right) \xi\left(u^{2}-\xi^{2}\right)} \log ^{2}(1-\bar{\omega} \xi) \\
& +(\bar{\omega} \leftrightarrow-\bar{\omega}), \\
& C_{1}^{S, g}(u \bar{\omega}, \xi \bar{\omega})=\left(\sum_{q} e_{q}^{2}\right) \frac{1}{2} \frac{u^{2}}{u^{2}-\xi^{2}}\left[\frac{4-4 \bar{\omega} u+\bar{\omega}^{2} u^{2}-\bar{\omega}^{2} \xi^{2}}{\bar{\omega}^{2}\left(u^{2}-\xi^{2}\right)} \log (1-\bar{\omega} u)\right. \\
& -\frac{2-2 \bar{\omega} u+\bar{\omega}^{2} u^{2}-\bar{\omega}^{2} \xi^{2}}{2 \bar{\omega}^{2}\left(u^{2}-\xi^{2}\right)} \log ^{2}(1-\bar{\omega} u) \\
& +\frac{(1-\bar{\omega} \xi)\left(\bar{\omega} u^{2}-4 \xi-\bar{\omega} \xi^{2}\right)}{\bar{\omega}^{2} \xi\left(u^{2}-\xi^{2}\right)} \log (1-\bar{\omega} \xi) \\
& \left.-\frac{(1-\bar{\omega} \xi)\left(\bar{\omega} u^{2}-2 \xi-\bar{\omega} \xi^{2}\right)}{2 \bar{\omega}^{2} \xi\left(u^{2}-\xi^{2}\right)} \log ^{2}(1-\bar{\omega} \xi)\right] \\
& +(\bar{\omega} \leftrightarrow-\bar{\omega}), \\
& C_{1}^{A, q}(u \bar{\omega}, \xi \bar{\omega})=-\frac{9}{2} \frac{u \bar{\omega}}{1-u \bar{\omega}}+\frac{u\left(\bar{\omega}^{2} u^{2}+3 \bar{\omega}^{2} \xi^{2}-2 \bar{\omega} u-2\right)}{2 \bar{\omega}(1-\bar{\omega} u)\left(u^{2}-\xi^{2}\right)} \log (1-\bar{\omega} u) \\
& +\frac{u\left(1+\bar{\omega}^{2} u^{2}-2 \bar{\omega}^{2} \xi^{2}\right)}{2 \bar{\omega}(1-\bar{\omega} u)\left(u^{2}-\xi^{2}\right)} \log ^{2}(1-\bar{\omega} u) \\
& +\frac{u(1-\bar{\omega} \xi)\left(1+2 \bar{\omega}^{2} u^{2}+3 \bar{\omega} \xi\right)}{\bar{\omega}\left(1-\bar{\omega}^{2} u^{2}\right)\left(u^{2}-\xi^{2}\right)} \log (1-\bar{\omega} \xi) \\
& -\frac{u(1-\bar{\omega} \xi)\left(1+\bar{\omega}^{2} u^{2}+2 \bar{\omega} \xi\right)}{2 \bar{\omega}\left(1-\bar{\omega}^{2} u^{2}\right)\left(u^{2}-\xi^{2}\right)} \log ^{2}(1-\bar{\omega} \xi) \\
& -(\bar{\omega} \leftrightarrow-\bar{\omega}) \text {, } \\
& C_{1}^{A, g}(u \bar{\omega}, \xi \bar{\omega})=\left(\sum_{q} e_{q}^{2}\right) \frac{1}{2} \frac{u^{2}}{u^{2}-\xi^{2}}\left[\frac{\left(3 \bar{\omega} u^{2}+\bar{\omega} \xi^{2}-4 u\right)}{\bar{\omega}\left(u^{2}-\xi^{2}\right)} \log (1-\bar{\omega} u)\right. \\
& +\frac{2 u-\bar{\omega} u^{2}-\bar{\omega} \xi^{2}}{2 \bar{\omega}\left(u^{2}-\xi^{2}\right)} \log ^{2}(1-\bar{\omega} u) \\
& \left.+\frac{4 u(1-\bar{\omega} \xi)}{\bar{\omega}\left(u^{2}-\xi^{2}\right)} \log (1-\bar{\omega} \xi)-\frac{u(1-\bar{\omega} \xi)}{\bar{\omega}\left(u^{2}-\xi^{2}\right)} \log ^{2}(1-\bar{\omega} \xi)\right] \\
& -(\bar{\omega} \leftrightarrow-\bar{\omega}) \text {, } \\
& C_{1}^{L, q}(u \bar{\omega}, \xi \bar{\omega})=\frac{-4 u}{\bar{\omega}\left(u^{2}-\xi^{2}\right)} \log (1-\bar{\omega} u)+\frac{4 u^{2}}{\bar{\omega} u^{2} \xi-\bar{\omega} \xi^{3}} \log (1-\bar{\omega} \xi) \\
& +(\bar{\omega} \leftrightarrow-\bar{\omega}) \\
& C_{1}^{L, g}(u \bar{\omega}, \xi \bar{\omega})=\left(\sum_{q} e_{q}^{2}\right) \frac{1}{2} \frac{u^{2}}{u^{2}-\xi^{2}}\left[\frac{8(1-\bar{\omega} u)}{\bar{\omega}^{2}\left(u^{2}-\xi^{2}\right)} \log (1-\bar{\omega} u)\right. \\
& \left.+\frac{4\left(\bar{\omega} u^{2}-2 \xi+\bar{\omega} \xi^{2}\right)}{\bar{\omega}^{2} \xi\left(u^{2}-\xi^{2}\right)} \log (1-\bar{\omega} \xi)\right]+(\bar{\omega} \leftrightarrow-\bar{\omega}) .
\end{aligned}
$$

The above expressions for the transverse and antisymmetric coefficient functions agree with the results presented in [8] and [9]. In the forward limit $\xi=0$ all coefficients coincide 
with the well-known one-loop corrections to the $\overline{\mathrm{MS}}$ Wilson coefficients in deep-inelastic scattering [19, 20] for the $F_{1}, g_{1}$ and $F_{L}$ structure functions. As noted already in [8], the transverse and antisymmetric coefficient functions are regular in the limit $Q^{\prime 2} \rightarrow 0$, i.e. $\bar{\omega} \xi \rightarrow 1$. After multiplying by the longitudinal photon polarization vectors $\epsilon_{L \mu}^{*}\left(q^{\prime}\right)$ and $\epsilon_{L \nu}(q)$, the complete longitudinal amplitude

$$
\begin{aligned}
& \epsilon_{L \mu}^{*}\left(q^{\prime}\right) \Pi_{\mu \nu} \epsilon_{L \nu}(q)=\frac{1}{2}\left(1-\bar{\omega}^{2} \xi^{2}\right)^{1 / 2} \\
& {\left[\sum_{q} e_{q}^{2} \int_{-1}^{1} \frac{d u}{u} F_{q}\left(u, \xi ; \bar{Q}^{2}\right) a_{s} C_{1}^{L, q}(u \bar{\omega}, \xi \bar{\omega})+\int_{-1}^{1} \frac{d u}{u} G\left(u, \xi ; \bar{Q}^{2}\right) a_{s} C_{1}^{L, g}(u \bar{\omega}, \xi \bar{\omega})\right],}
\end{aligned}
$$

vanishes when one of the photons goes on shell.

A few comments are in order: to isolate the gluonic coefficients $C_{1}^{i, g}, i=S, A, L$ we found it advantageous to work in light-cone gauge $n \cdot A=0$. Here one can formally express the non-forward matrix element of the product of two gluon fields by a combination of non-forward parton distributions [1, 21]:

$$
\begin{aligned}
\left\langle P^{\prime}\left|A_{\rho}^{A}\left(-\frac{z}{2}\right) A_{\sigma}^{A}\left(\frac{z}{2}\right)\right| P\right\rangle_{z^{2}=0} & =\frac{1}{d-2}\left(-g_{\rho \sigma}\right)^{T} \int_{-1}^{1} d u \frac{u G\left(u, \xi ; \mu^{2}\right)}{(u-\xi+i \epsilon)(u+\xi-i \epsilon)} e^{-i u \bar{P} \cdot z} \\
& -\frac{1}{d-2} i \epsilon_{\rho \sigma \alpha \beta} \frac{n^{\alpha} n^{* \beta}}{n \cdot n^{*}} \int_{-1}^{1} d u \frac{u \Delta G\left(u, \xi ; \mu^{2}\right)}{(u-\xi+i \epsilon)(u+\xi-i \epsilon)} e^{-i u \bar{P} \cdot z} .
\end{aligned}
$$

The factor $\frac{1}{d-2}$ in the second line arises from the relation between the product of the gluon field strength tensor and its dual, and the polarized gluon distribution (see e.g. [22]). Here a product of two epsilon tensors arises which can be expressed by a determinant of metric tensors in $d$ dimensions. Although within such a scheme unpolarized and polarized contributions in Eq.(10) are treated symmetrically, the factor $\frac{1}{d-2}$ is customary set to its $d=4$ value in the current literature [23]. As different choices correspond merely to different factorization schemes], the result for $C_{1}^{A, g}$ presented in (8) has been calculated according to the standard convention.

In the case of $C_{1}^{A, q}$ there are well-know complications [24] related to the 'tHooftVeltman definition of the $\gamma_{5}$ matrix in $d$ dimensions [25]. In order to avoid a non-vanishing two-loop anomalous dimension of the flavor non-singlet axial current operator we have followed Ref. 26] and imposed an additional, finite renormalization of the twist-2 axial string operator $A\left(-\frac{z}{2}, \frac{z}{2}\right)=\bar{q}\left(-\frac{z}{2}\right)\left[-\frac{z}{2} ; \frac{z}{2}\right] \gamma_{5} \hat{z} q\left(\frac{z}{2}\right)$. It can be derived from the condition 24] that the renormalized vertices of the operators $A\left(-\frac{z}{2}, \frac{z}{2}\right)$ and $V\left(-\frac{z}{2}, \frac{z}{2}\right)=\bar{q}\left(-\frac{z}{2}\right)\left[-\frac{z}{2} ; \frac{z}{2}\right] \hat{z} q\left(\frac{z}{2}\right)$ coincide up to the $\gamma_{5}$ matrix:

$$
\tilde{Z}_{5}^{n s} \otimes R_{\overline{\mathrm{MS}}}\langle\bar{\psi} A \psi\rangle=\gamma_{5} R_{\overline{\mathrm{MS}}}\langle\bar{\psi} V \psi\rangle
$$

${ }^{\S}$ We are grateful to W. Vogelsang for discussions about this point. 
Here $R_{\overline{\mathrm{MS}}}$ denotes the R-operation, i.e. the subtraction of the UV singularity in the $\overline{\mathrm{MS}}$ scheme. The symbol $\otimes$ in the above equation stands for the convolution of the kernel $\tilde{Z}_{5}^{n s}$ with the operator $A$ according to:

$$
\tilde{Z}_{5}^{n s} \otimes A=\int_{0}^{1} d \lambda \int_{0}^{\bar{\lambda}} d \rho \tilde{Z}_{5}^{n s} A\left(-\frac{z}{2}(1-2 \lambda), \frac{z}{2}(1-2 \rho)\right)
$$

with $\bar{\lambda}=1-\lambda$, and to one loop accuracy,

$$
\tilde{Z}_{5}^{n s}=\delta(\bar{\lambda}) \delta(\bar{\rho})+a_{s}\left(-8 C_{F}\right) .
$$

The condition (11) is clearly inappropriate in the case of the flavor-singlet axial current, which acquires an anomalous dimension at the two-loop level due to the axial anomaly [26, 27]. We have therefore undone the effect of (13) in the flavor-singlet coefficient $C_{1}^{A, q}$ by subtracting the term proportional to $\bar{\omega}$ in the DVCS amplitude resulting from $\tilde{Z}_{5}^{n s}$. Subsequently we have replaced it by a contribution which follows from the application of the renormalization constant of the flavor singlet axial current operator. This has been determined to two-loop accuracy in Ref. [24] from the requirement that the anomaly equation is preserved at higher orders. After this correction has been incorporated it turns out that the one-loop coefficients $C_{1}^{A, q}$ are the same in the flavor singlet and non-singlet channels. Different choices of normalizations of the flavor singlet axial current can again be related to different factorization schemes. A physical quantity, like the antisymmetric part of the deeply-virtual Compton scattering amplitude, does not depend on the choice of $\tilde{Z}_{5}^{s}$, provided one consistently defines the NLO evolution equations $\square$.

It is perhaps worthwhile to note that at this point one can fully appreciate the power of the conformal approach [9], which allows to determine the coefficient functions (8) from the well-known one-loop Wilson coefficients in the forward case.

We have presented a calculation of NLO corrections to the deeply-virtual Compton scattering amplitude. An analysis of future experimental data requires an analytical continuation of Eq.(6) to the physical domain $\bar{\omega}>1$, and the inclusion of NLO effects in the evolution of non-forward parton densities. The latter are known at present only for the non-singlet sector [28]. However, even before a complete NLO analysis is completed, it will be interesting to see, using perhaps some phenomenological model for non-forward parton distributions [10, 6] to which extent corrections discussed in this paper modify the leading-order results.

\section{Acknowledgments}

This work was supported in part by BMBF and by KBN grant 2 P03B 065 10. E.S. thanks Deutsche Forschungsgemeinschaft (DFG) for financial support. We thank Dieter Müller for pointing out an error in Eq.(8).

\footnotetext{
IWe thank S. Forte for discussions about this point.

"The phenomenological parametrization used in ref. [6] has been proposed by A. Radyushkin
} 


\section{References}

[1] B. Badelek, J. Bartels, N. Brook, A. De Roeck, A. Vogt, T. Gehrmann, A.D. Martin, M. Lancaster, J. Phys. G22, 815 (1996).

[2] J.C. Collins, D.E.. Soper and G. Sterman, in "Perturbative Quantum Chromodynamics", ed. by A.H. Mueller (World Scientific, Singapore, 1989) p. 1.

[3] X. Ji, Phys. Rev. D55, 7114 (1997);

X. Ji, Phys. Rev. Lett. 78, 610 (1997).

[4] A. Radyushkin, Phys. Lett. B380, 417 (1996);

A. Radyushkin, Phys. Lett. B385, 333 (1996);

A. Radyushkin, Phys. Rev. D56, 5524 (1997).

[5] J. Collins, L. Frankfurt and M. Strikman, Phys. Rev. D56, 2982 (1997).

[6] L. Mankiewicz, G. Piller and T. Weigl, Hard Exclusive Meson Production and Nonforward Parton Distributions, hep-ph/9711227 (1997).

[7] D. Müller, Restricted conformal invariance in $Q C D$ and its predictive power for virtual photon processes, hep-ph/9704406 (1997).

[8] X. Ji, J. Osborne, One loop QCD corrections to deeply virtual Compton scattering: the parton helicity-independent case, hep-ph/9707254 (1997).

[9] A.V. Belitsky, D. Müller, Predictions from conformal algebra for the deeply-virtual compton scattering, hep-ph/9709379 (1997).

[10] X. Ji, W. Melnitchouk, X. Song, Phys. Rev. D56, 5511 (1997);

V. Petrov, P. Pobylitsa, M. Polyakov, I. Börning, K. Goeke and C. Weiss, Off-forward quark distributions of the nucleon in the large $N_{C}$ limit, hep-ph/9710270, (1997).

[11] Proceedings of the International Workshop on Virtual Compton Scattering, 1996, Clermont-ferrand, France, V. Breton (ed.).

[12] M. Diehl, T. Gousset, B. Pire, J. P. Ralston, Testing the handbag contribution to exclusive virtual Compton scattering, hep-ph/9706344, (1997).

[13] K. Watanabe, Prog. Theor. Phys. 67, 1834 (1982).

[14] F. M. Dittes, D. Müller, D. Robaschik, B. Geyer, and J. Horejsi, Phys. Lett. B209, 325 (1988).

[15] Z. Chen, Non-forward and Unequal Mass Virtual Compton Scattering, hepph/9705279 (1997). 
[16] S. G. Gorishny, S. A. Larin and F. V. Tkachov, Phys. Lett. B124,217 (1983); S. G. Gorishny and S. A. Larin, Nucl. Phys. B283, 452 (1987).

[17] C. Itzykson, J.-B. Zuber, Quantum Field Theory (McGraw Hill, New York, 1986).

[18] I. Balitskii and V. Braun, Nucl. Phys. B311, 541 (1988/89).

[19] W.A. Bardeen, A.J. Buras, D.W. Duke, and T. Muta, Phys. Rev. D18, 3998 (1978).

[20] J. Kodaira, S. Matsuda, T. Muta, K. Sasaki, T. Uematsu, Phys.Rev. D20 627 (1979);

J. Kodaira, S. Matsuda, K. Sasaki, T. Uematsu, Nucl.Phys. B159, 99 (1979);

G. Altarelli, R.K. Ellis, G. Martinelli, Nucl. Phys. B157, 461 (1979);

B. Humpert and W.L. van Neerven, Nucl. Phys. B184, 225 (1981);

G.T. Bodwin and J. Qiu, Phys. Rev. D41, 2755 (1990);

W.Vogelsang, Z.Phys. C50, 275 (1991).

[21] X. Ji, Phys. Lett. B289,137 (1992).

[22] A. Saalfeld, G. Piller and L. Mankiewicz, Gluon polarization in nucleons, Z. Phys. C, in print, hep-ph/9708378.

[23] E.B. Zijlstra, W.L. van Neerven, Nucl. Phys. B417, 61 (1994), Erratum-ibid. B426, 245 (1994).

[24] S.A. Larin, Phys. Lett. B303, 113 (1993);

T.L. Trueman, Phys. Lett. B88, 331 (1979).

[25] G.'t Hooft and M. Veltman, Nucl.Phys.B 44, 189 (1972);

D. A. Akyeampong and R. Delborgo, Nuovo Cim. 17A, 578 (1973);

P. Breitenlohner and D. Maison, Comm.Math.Phys.52, 11 (1977).

[26] S.A. Larin, Phys.Lett. B334, 192 (1994).

[27] S.L. Adler, Phys.Rev. 177, 2426 (1969);

J.S. Bell and R. Jackiv, Nuovo Cim.60A, 47 (1969);

S.L. Adler and W. Bardeen, Phys.Rev.182, 1517 (1969).

[28] F.M. Dittes, A. Radyushkin, Phys. Lett. 134 B, 359 (1984);

M.H. Sarmadi, Phys. Lett. 143B, 471 (1984);

G.R. Katz, Phys. Rev. D31, 652 (1985);

S.V. Mikhailov, A.V. Radyushkin, Nucl. Phys. B254, 89 (1985). 